\title{
The Impact of Autonomic Dysreflexia on Blood Flow and Skin Response in Individuals with Spinal Cord Injury
}

\author{
J. C. Ramella-Roman and J. M. Hidler \\ Department of Biomedical Engineering, The Catholic University of America, 620 Michigan Avenue, \\ N.E. Washington, DC 20064, USA \\ Correspondence should be addressed to J. C. Ramella-Roman, ramella@cua.edu
}

Received 5 March 2008; Accepted 1 June 2008

Recommended by Stoyan Tanev

\begin{abstract}
Autonomic dysreflexia $(\mathrm{AD})$ is an inappropriate response of the sympathetic nervous system that commonly occurs when individuals with spinal cord injury (SCI), at or above the sixth thoracic (T6) vertebra, are subjected to a noxious stimulus below the level of injury. An AD event can be put into motion by something as simple as an ingrown toenail or a full bladder, with symptoms ranging from headache, high blood pressure, and even stroke. We have characterized the onset of AD and resulting autonomic events in an individual with SCI using a fiberoptic-based probe. Two probes were located above and below the injury level, on the subjects forearm and thigh, respectively, and were connected to a dual channel spectrophotometer. Oxygen saturation was calculated using the reflectance spectra and an algorithm based on melanin and hemoglobin absorption. We noticed that during an $\mathrm{AD}$ event the amount of oxygen in the skin below the injury level dropped by as much as $40 \%$, while above the injury level, skin oxygenation remained constant. In addition, we found that the level of skin perspiration below the level of injury increased significantly. We hypothesize that the combination of AD-related ischemia with pressure-related ischemia and increased perspiration places individuals with spinal cord injury level at $\mathrm{T} 6$ or above at an elevated risk for developing a pressure sore below the injury site.
\end{abstract}

Copyright (c) 2008 J. C. Ramella-Roman and J. M. Hidler. This is an open access article distributed under the Creative Commons Attribution License, which permits unrestricted use, distribution, and reproduction in any medium, provided the original work is properly cited.

\section{INTRODUCTION}

Autonomic dysreflexia (AD) is an inappropriate response of the sympathetic nervous system in individuals with spinal cord injuries at or above the sixth thoracic vertebra (T6). Some common symptoms of AD include excessive sweating, pilomotor erection of skin hairs, headache, hypertension, blurring of vision, and more rarely convulsion, loss of consciousness, cerebral hemorrhage, and death [1]. An AD event is often induced by a noxious stimulus below the level of injury, such as a full bladder, an ingrown nail, or a pressure sore.

The autonomic nervous system is responsible for maintaining the body's homeostasis via the parasympathetic nervous system and the sympathetic nervous system. These two systems have in most cases complementary roles and exist in two different locations of the central nervous system. In patients with $\mathrm{T} 6$ injuries or higher, the communication between the parasympathetic nervous system and the sympathetic nervous system is interrupted, so that the stimulation of one branch no longer corresponds to the suppression of the other, resulting in the negative feedback loop of both systems no longer working appropriately. A noxious stimulus below $\mathrm{T} 6$ can cause a sympathetic response resulting in vasoconstriction, headaches, pallor, and chills below the injury level. Vasoconstriction produces a rise in blood pressure and consequent reflex bradycardia through the baroreceptor reflex $[1,2]$. Because of disruptions in descending neural pathways, the parasympathetic nervous system is unable to send or receive information regarding the status of the body below the injury level, but tries to maintain the body's homeostasis by slowing down the heart rate. As a result, above the injury level the patient will experience a vagal response with vasodilation and bradycardia. An $\mathrm{AD}$ episode can normally be stopped quickly by removing the painful stimulus that triggered it; however, if no action is taken, the individual will experience elevating levels of symptoms. $\mathrm{AD}$ has been the focus of many studies for its 
cardiovascular effects [3], however, no study to date has evaluated its effect on pressure sore formation. We believe that the circulatory dysfunction caused by $\mathrm{AD}$ (hypoxia and ischemia) can negatively impact the health of the skin contributing to the formation of skin ulcers.

Skin hypoxia is often referred to as a one of the main causes of skin damage $[4,5]$. Several studies have shown that hypoxic wounds do not heal as quickly as well oxygenated wounds [5], forcing an investigation on the pathophysiological impact of hypoxia on healthy skin [6]. Previous studies have shown that hypoxia leads to tissue angiogenesis, vascular leakage, and consequently to skin deterioration [7]. A mediator in these alterations is the vascular permeability factor [8] (VPF, also known as vascular endothelial growth factor (VEGF)). Steinbrech et al. [9] have shown a time-dependent change in levels of VEGF and type II collagen under hypoxic condition. Levels of VEGF mRNA in normal human dermal fibroblast increased by $140 \%$ after 6 hours of hypoxia. Likewise, collagen mRNA expression increased by $170 \%$ after 24 hours of hypoxia. Finally Yamanaka and Ishikawa [5] showed that decreasing the oxygen tension to $2 \%$ increased mRNA of type I collagen from cultured human skin. Recently Dalton et al. [6] have shown each of these factors contribute to dermal failure and the formation of leg ulcers. Ischemia is also a recognized risk factor in skin ulcer formation. Thorfinn et al. $[10,11]$ have shown that sitting on a hard surface reduces perfusion to a biological zero.

Several studies used reactive hyperemia [12-14] as a way to measure perfusion response to load and skin stress. Herrman et al. [15] used a rat model to show that long-term ischemia ( 5 hours) due to elevated surface pressure causes a persistent hyperemic response, suggesting a compromise in the vasodilator mechanisms. In fact, persistent hyperemia is considered stage I skin breakdown in the National Pressure Ulcer Advisory Panel (NPUAP) definitions. In a related study by Thorfinn et al. [11], it was found that through the use of laser doppler flowmetry (LDF), perfusion in the skin over the ischial tuberosities of individuals with SCI after short and long periods of loading was higher than in normal individuals although the initial hyperemic response was significantly lower for SCI patients. Munstoe et al. $[16,17]$ have studied the role of ischemic-reperfusion injury in chronic wounds and have pointed out that the related inflammatory mechanism caused by a release of free radicals could lead to pressure ulcers. Finally, recent studies [18] on animal models have shown that the onset of anoxia on an already ischemic environment may cause a restructuring of the capillary bed with a permanent increase in skin perfusion. Stover et al. $[19,20]$ have shown that AD is a possible cause of skin restructuring. In studying skin thickening in SCI patients with and without AD symptoms it was shown that $80 \%$ of individuals with severe AD (grade 3 ) developed skin thickening compared to the $22 \%$ of patients without $\mathrm{AD}$ symptoms. The reduction of oxygenated flow coming from the femoral artery, characteristic of autonomic dysreflexia, is likely to exacerbate the ischemic hypoxia due to pressure. AD also causes increased perspiration that has been linked to skin ulcer formation [21]. The goal of this study was to quantify blood flow rate, skin oxygenation levels, the amount of skin perspiration, and other autonomically related events during an episode of autonomic dysreflexia in an individual with spinal cord injury. We hypothesize that during $\mathrm{AD}$, the blood flow rate below the injury level will drop significantly, translating into meaningful decreases in skin oxygen levels. Additionally, we hypothesize that during $\mathrm{AD}$, the amount of skin perspiration will increase below the injury level.

\section{MATERIAL AND METHODS}

Our preliminary measurements were made on an individual with a T6 level injury classified as ASIA A on the American Spinal Injury Association sensory and motor impairment scale. As such, this individual had no sensation or volitional movement below the T6 injury level. Five different diagnostic modalities were measured: blood pressure, lower limb temperature, femoral artery blood flow velocity, pulse oximetry in the right thumb and right toe, and oxygen saturation in the forearm skin (above injury level), and thigh skin (below injury level). Measurements were first taken from the subject 15 minutes after the subject emptied his bladder. The subject then began consuming water, slowly filling his bladder. The test was interrupted when the subject acknowledged persistent symptoms of $\mathrm{AD}$ resulting from a full bladder, while his blood pressure remained under 150/100 mm Hg. All measurements were conducted in 5 minutes intervals apart from skin oxygenation that was measured automatically every 30 seconds. Systolic and diastolic pressures were measured with an electronic blood pressure measuring device (Jobar International, China). A thermocouple attached to the patients left leg measured surface temperature. The femoral artery blood velocity was measured with an ultrasound probe (HP Sonos 2000, Palo Alto CA). Measurement of oxygen saturation on the patients thumb and toe were done with a portable pulse oximeter (Digit, Smiths Medical, Waukesha, Wis, USA). Finally, skin oxygenation was measured spectroscopically.

Our spectroscopic technique measures local oxygen saturation $\mathrm{StO}_{2}$ in the skin microcirculation and differs from pulse oximetry which measures oxygen saturation in arterial blood only. Our experimental apparatus consisted of two custom-made optical probes constructed with two optical fibers (Figure 1), and a dual channel spectrophotometer (Ocean Optics, Dunedin, Fla, USA).

Fiber diameter and separation were chosen in order to obtain a shallow probing depth $(<1.5 \mathrm{~mm})$. This ensured correct measurement of superficial skin. Two $0.6 \mathrm{~mm}$ diameter multimode fibers (Ocean Optics, Dunedin, Fla, USA) were positioned at $2.2 \mathrm{~mm}$ center to center and were supported by a Delrin casing. One fiber was used as the light source and was attached to a tungsten halogen lamp (Ocean Optics, Dunedin, Fla, USA); the second fiber was used as a detector and was connected to one channel of the spectrophotometer (Ocean Optics, Dunedin, Fla, USA). Finally the device was tested on a custom-made reflectance standard described by Moffitt et al. [22]. Similar probes have been used recently to measure tumor oxygenation in vivo [23], as well as optical 


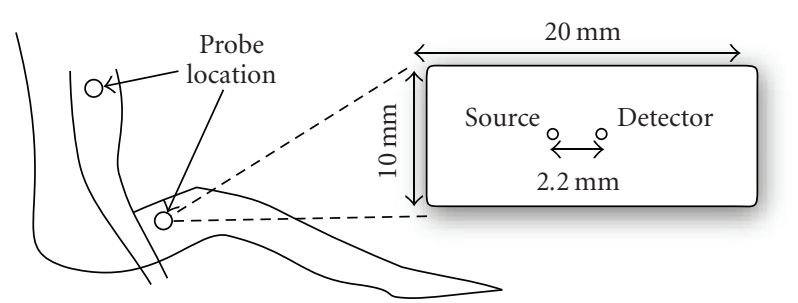

Figure 1: Measurement location of the fiber optic probe used in the skin oxygen saturation measurements. The fiber optics were enclosed in a black Delrin case with a fiber to fiber separation of $2.2 \mathrm{~mm}$, both fibers were $0.6 \mathrm{~mm}$ in diameter.

properties of scattering biological tissues [24, 25], and are ideal for measurements where the penetration depth is low. The probing depth of our system was assessed with the aid of Monte Carlo simulations of light propagation into skin. Figure 2 shows the results of a simulation conducted with 3 million photons.

Skin was represented by two layers of different optical properties. The top most layer represented the epidermis and was $60 \mu \mathrm{m}$ in thickness $\left(\mu_{a}=35 \mathrm{~cm}^{-1}, \mu_{s}=450 \mathrm{~cm}^{-1}\right.$, and $g=0.78$ at $540 \mathrm{~nm}$ ); the second layer, representing the dermis, was semiinfinite $\left(\mu_{a}=5 \mathrm{~cm}^{-1}, \mu_{s}=450 \mathrm{~cm}^{-1}\right.$, and $g=0.81$ at $540 \mathrm{~nm}$ ); the skin optical properties for each layer were from [26] by van Gemert et al. The Monte Carlo program followed the general structure of MCML [27] and was able to handle layers but did not consider Fresnel reflections at the interfaces. Photons were launched by a source fiber $600 \mu \mathrm{m}$ in diameter, $\mathrm{NA}=0.39$, and collected by an identical detector fiber at $0.22 \mathrm{~mm}$ distance from the source. The propagation media was divided in square bins $10 \mu \mathrm{m} \times 10 \mu \mathrm{m}$ in size, initially all the bins were set to a zero value. Only the trajectories of the photons leaving the source fiber and reaching the detector fiber were tracked. Each time a photon reached a location in the media the value of the corresponding bin was increased by one fold (see insert in Figure 2).

Moore et al. [28] have measured dermis thickness using ultrasound both for able-bodied individuals as well as individuals with systemic sclerosis showing no significant difference among the two populations, dermis thickness in the right thigh was $1.379 \pm 0.217 \mathrm{~mm}$ for controls and $0.8 \pm$ $0.13 \mathrm{~mm}$ in the upper arm. In light of these measurements and looking at Figure 2, we can note that the photons traveling from the source to the collector fiber reached depths as far as $1.5 \mathrm{~mm}$, spanning the full length of a normal epidermis and dermis $[26,28]$. From this simple simulation we can assume that the probe design is suitable to measure superficial skin hemodynamics.

\subsection{Phantom measurements}

Calibration of the fiber optic probe was accomplished with an optical phantom, whose optical properties had been characterized as described in the paper by Moffitt et al. [22]. From the calibrated optical properties, and using Farrells equation [29], we calculated the total reflectance captured by a fiber optic layout with $2.2 \mathrm{~mm}$ center-tocenter fiber separation (thin line). Finally, we experimentally measured the standard reflectance using the fiber probe and spectrometer as discussed in the previous section. The fiber tip/phantom interface was matched using a water droplet, and a 99\% Spectralon reflectance standard was used to normalize the experimental results, both experimental and calibrated values are shown in Figure 3.

\subsection{Analysis of spectral data}

Two different algorithms yielding similar results were used to model the reflectance spectra acquired with the spectrophotometer. The first algorithm is described by Kollias and Baqer [25] and uses the curve of absorbance of skin, which is simply the logarithm of the ratio of the skin diffused reflectance to a reflectance standard (Spectralon standard 99\% reflectance). The total absorbance curve of skin is corrected for melanin absorption by subtracting its contribution from the general data. Skin pigmentation is approximated as the slope of a fitted straight line between the values of absorbance at $620 \mathrm{~nm}$ and $720 \mathrm{~nm}$, the absorbance curve of melanin decreasing monotonically between $600 \mathrm{~nm}$ and $750 \mathrm{~nm}$. Oxygen saturation is calculated by using tabulated absorption curves of oxygenated and deoxygenated hemoglobin to fit the experimental data in the range $550 \mathrm{~nm}$ to $580 \mathrm{~nm}$. In this range both curves exhibit local maxima [30].

The second algorithm used to model reflectance spectra is based on the Farrells equation and has been used by Bargo et al. [23, 24]. Tabulated skin chromophores spectra [30, 31] are used to model the skin absorption $\mu_{a}$ and reduced scattering coefficient $\mu_{s}^{\prime}$. The model considers the effect of the absorption of oxygenated hemoglobin $\mu_{a}^{\text {oxy }}$, deoxygenated hemoglobin $\mu_{a}^{\text {deoxy }}$, water $\mu_{a}^{\text {water }}$, and melanin $\mu_{a}^{\text {melanin }}$. The scattering coefficient of skin is modeled as a combination of Mie $(M)$ and Rayleigh $(R)$ scatterers [32]:

$$
\begin{aligned}
M & =4.5910^{3} \mathrm{~nm}^{-0.913}\left[\mathrm{~cm}^{-1}\right], \\
R & =1.7410^{12} \mathrm{~nm}^{-4}\left[\mathrm{~cm}^{-1}\right], \\
\mu_{s}^{\prime} & =a(M+R)\left[\mathrm{cm}^{-1}\right] .
\end{aligned}
$$

The total absorption in the skin is given by

$$
\mu_{a}=B\left(\mathrm{StO}_{2} \mu_{a}^{\text {oxy }}+\left(1-\mathrm{StO}_{2} \mu_{a}^{\text {deoxy }}\right)\right)+W \mu_{a}^{\text {water }}\left[\mathrm{cm}^{-1}\right] .
$$

The calculated optical coefficients $\mu_{a}$ and $\mu_{s}^{\prime}$ are used in combination with fiber separation and interface index of reflection in the Farrells equation to calculate total reflectance ( $R_{\text {Farrel }}$ ) from skin in the range of wavelengths of interest $(500-700 \mathrm{~nm})$. The impact of melanin is finally added to the model using a simple Beer-Lambert law as shown below:

$$
\operatorname{Total}_{R}=C e^{-N \mu_{a}^{\text {Melanin }} 2 L_{\text {epidermis }}} R_{\text {Farrel }},
$$

where $\mu_{a}^{\text {melanin }}=6.610^{11} \mathrm{~nm}^{-3.33}$ [32] and the photon trajectory in and out of the epidermis is taken into account by 


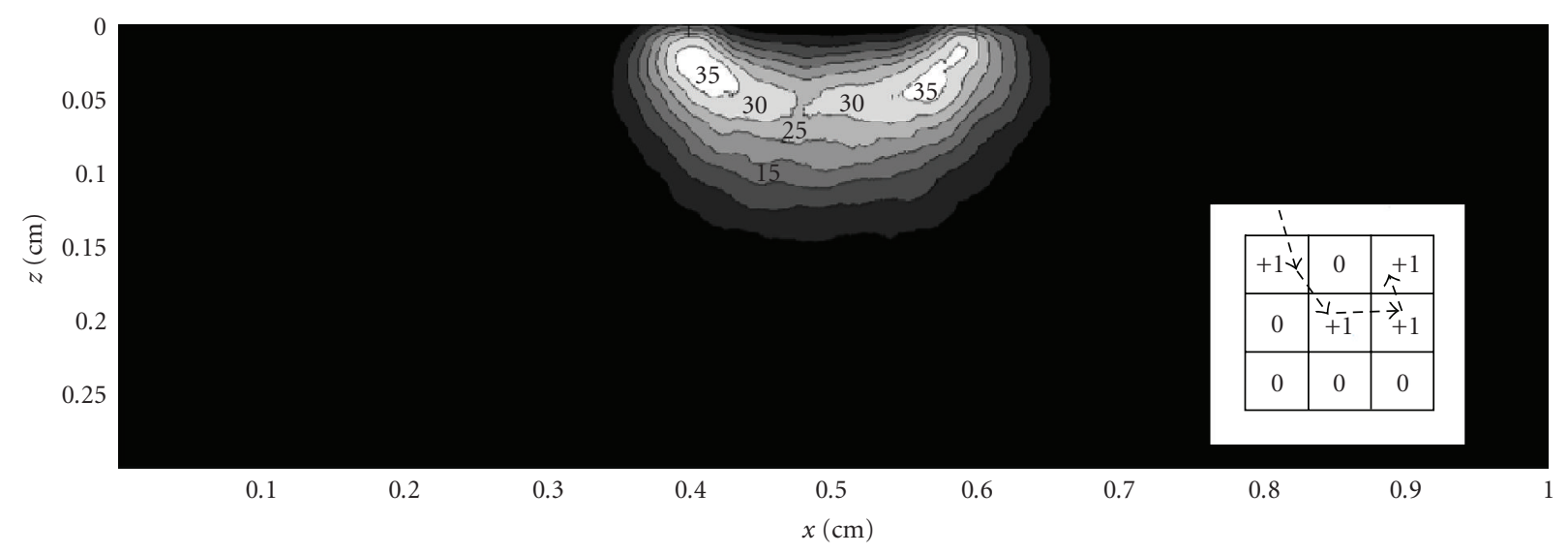

Figure 2: Monte Carlo simulation of light propagation in tissue. The different contours represent the number of photons reaching different depths (bin). In the insert, a pictorial description of our approach, every time a photon reaches a particular location the bin values are increased (+1). The graphs shows that photons will reach depths as far as $1.2 \mathrm{~mm}$ and still be able to return to the collection fiber. An averaging filter $(20 \times 20$ pixels in size $)$ was used to smooth the figure and to generate legible contours; the general trend shown in the graph did not change with the addition of the filter.

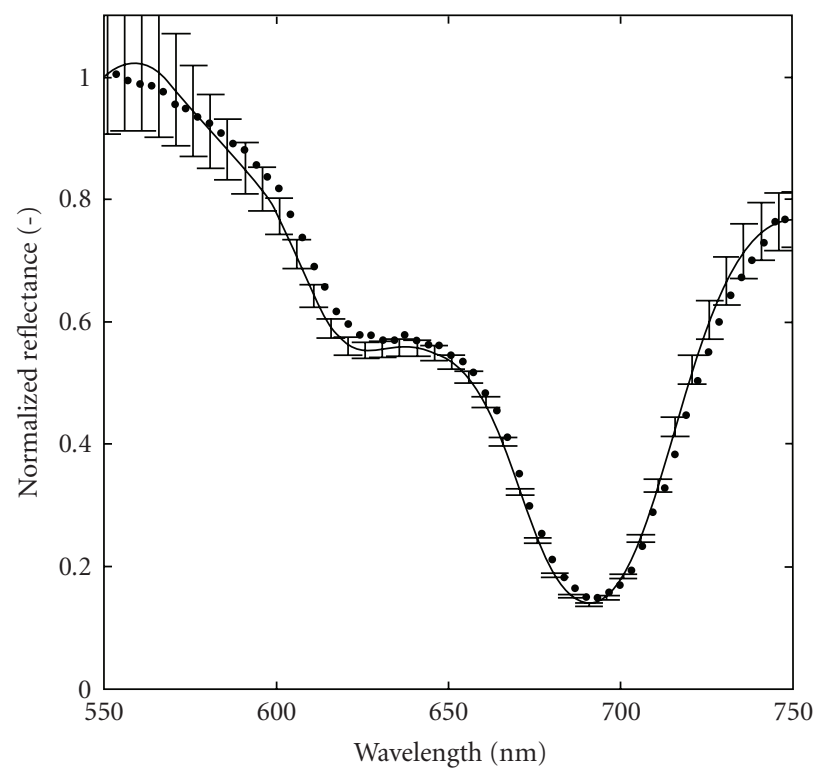

Figure 3: Calibration of the fiber optic probe. The solid symbols represent the values obtained experimentally with the fiber probe and the Ocean Optics spectrometer. The dashes represent the calculated reflectance for a set of experimentally measured optical properties. The optical properties of the standard had a larger margin of uncertainty at lower wavelengths as shown by the large standard deviation. The experimental results and theoretical findings were both normalized by the $550 \mathrm{~nm}$ reflectance.

multiplying the epidermis thickness by 2 . For these models we considered the epidermis to be $60 \mu \mathrm{m}$ in thickness. A least square mechanism is finally used to fit the theoretical results to the experimental data, fitting parameters are oxygen saturation $\left(\mathrm{StO}_{2}\right)$, water content $(W)$, blood fraction $(B)$, scattering fraction $(a)$, and melanin fraction $(N)$. A wavelength-independent parameter $C$ is used to account for

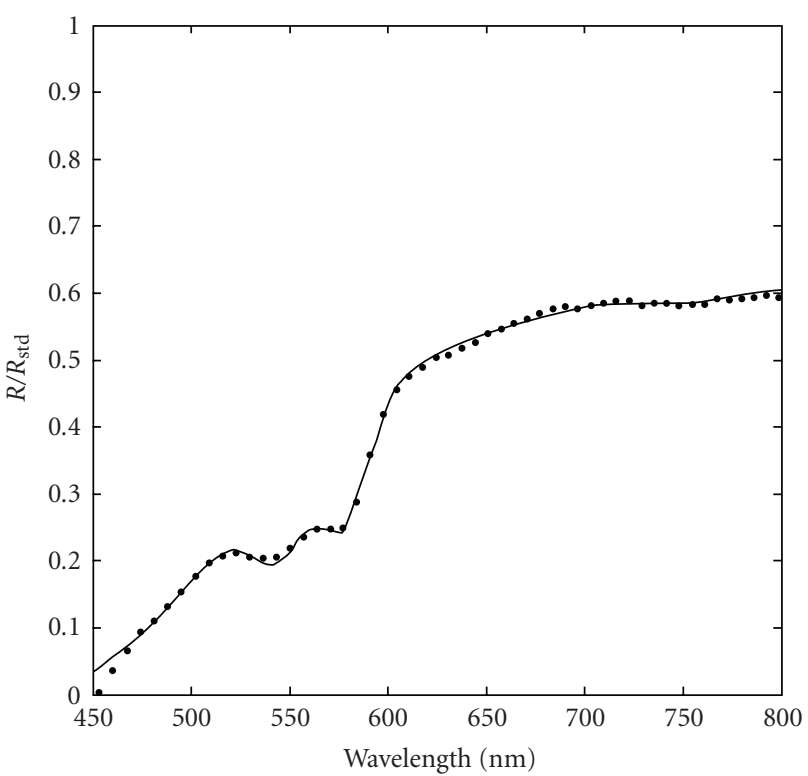

FIGURE 4: Normalized data (filled circles) and corresponding least square fit (line) obtained with the model by Bargo et al. Values of $\mathrm{StO}_{2}$ for this particular fit were 0.58 , the fraction of water was 0.65 , and the blood volume fraction was 0.0009 .

the effect of fiber collection efficiency [24]. This method has the advantage of obtaining information not only for oxygen saturation, but also for the skin components previously mentioned. These parameters can then be used to assess the quality and stability of the model. A typical least square fit obtained with this model is shown in Figure 4.

Both models yield similar results showing a decrease of oxygen saturation in the skin below the injury level at the onset of AD. Although the general temporal trend was identical for both algorithms, the numerical values of $\mathrm{StO}_{2}$ were at times different, up to $10 \%$. This difference 


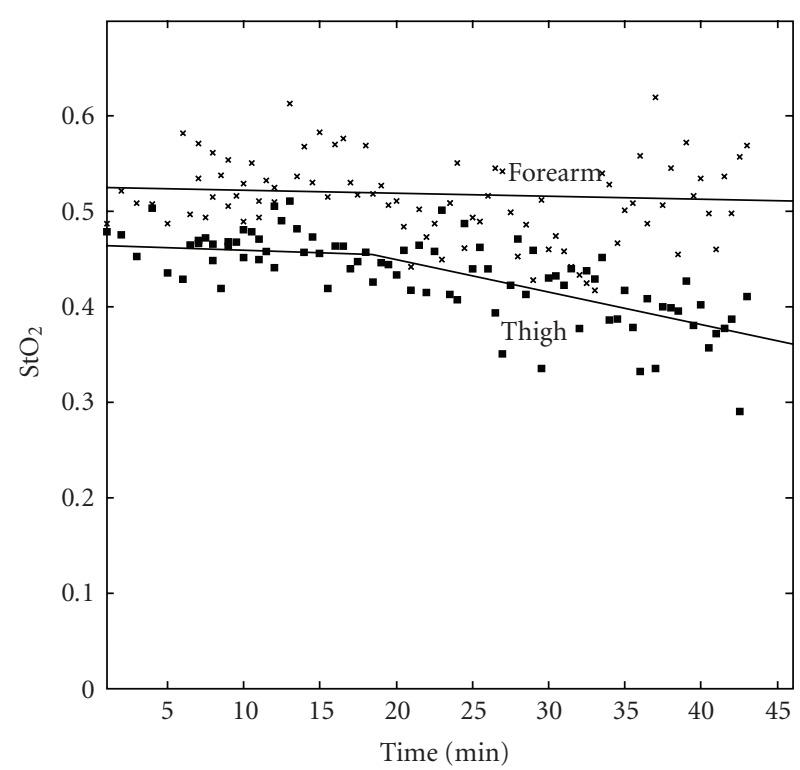

FIGURE 5: The effect of $\mathrm{AD}$ on the skin of an individual with complete SCI. The measurement on the forearm albeit noisy is stable around $\left(\mathrm{StO}_{2} \sim 52 \%\right)$, while the measurement on the thigh decreases over time going from $52 \%$ down to $\sim 37 \%$.

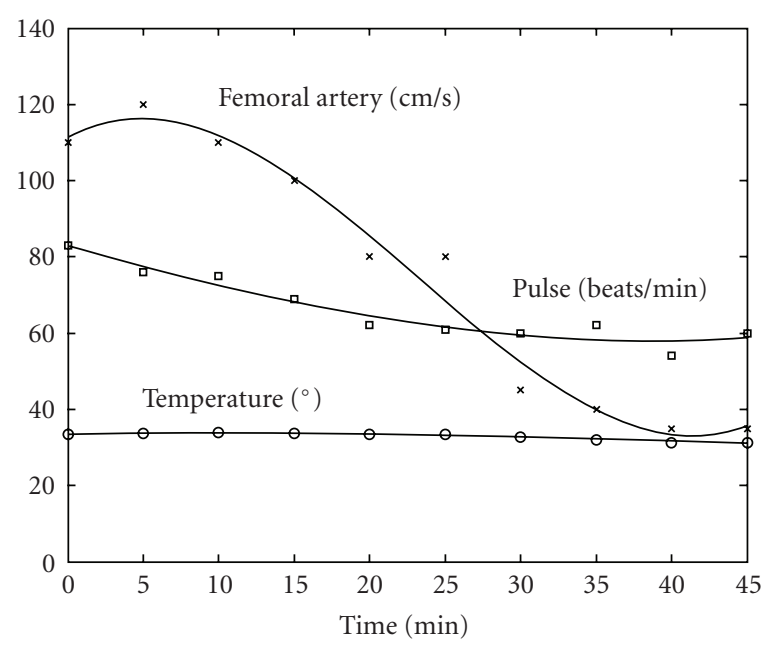

FIgURE 6: Measured modalities during an AD event, the lines 2nd order fits to the data.

can be minimized by changing the starting parameters of Bargo's algorithm. In this paper we will show the $\mathrm{StO}_{2}$ results obtained with the algorithm by Kollias et al.

\section{RESULTS}

Typical results obtained with the spectrophotometer and the fiber-optic probes over the experimental test session are shown in Figure 5. Two measurements were taken simultaneously on the subjects forearm and thigh using a dual channel spectrophotometer. $\mathrm{AD}$ causes a dilation of the vessel above the injury level and a constriction of the

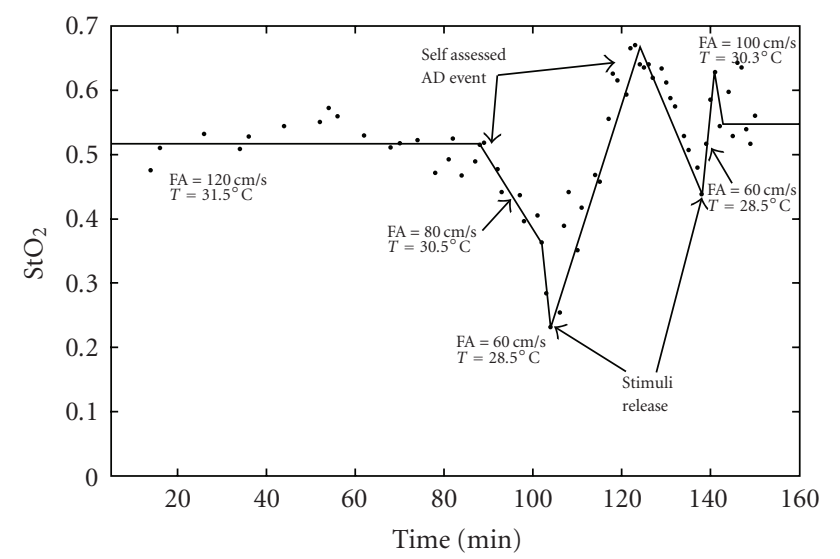

Figure 7: Two sequential AD events as detected by a SCI individual. At stimulus release oxygen saturation increases and overshoots to values above the norm. The patient relieved himself twice during this test at about 100 minutes and 140 minutes from the start of the test. Lines were manually drawn to pinpoint the $\mathrm{AD}$ events.

vessels below it. The purpose of this test was to show the effect of vessel constriction on the skin surface. Values of skin oxygenation $\left(\mathrm{StO}_{2}\right)$ were constant at the beginning of the measurement for the first 15 minutes. However, as the subject started experiencing $\mathrm{AD}$, oxygen saturation decreased quickly. Similar trends are observed in other diagnostic modalities (Figure 6).

The temperature in the subjects lower extremities decreased from $33^{\circ} \mathrm{C}$ to $31.2^{\circ} \mathrm{C}$ over the course of the experiment, while femoral artery systolic velocity dropped from $\sim 110 \mathrm{~cm} / \mathrm{sec}$ to $\sim 40 \mathrm{~cm} / \mathrm{sec}$. Interestingly, the pulse rate progressively slowed during the $\mathrm{AD}$ event, however we did not notice a significant increase in blood pressure as cited by the literature [21]. During the measurement the test subject described an increase in perspiration, and indeed an independent observation was made that the skin was noticeably moist to the touch; this is an important finding since humidity is considered a risk factor in the formation of skin ulcer, surprisingly analysis conducted with Bargo's model did not show any change in skin water content. Finally the pulse oximetry measurements on the thumb and toe remained constant at a $97 \%( \pm 1 \%$ ) level (not shown in graph). It is worth noting that while oxygen values in the main vessels remained constant, insufficient blood and oxygen reached the skin capillaries, possibly due to the vasoconstriction below T6.

During the entire experiment, we carefully monitored the well-being of the test subject, specifically the subjects systolic and diastolic blood pressure levels, as well as the subjects discomfort level. The first identification of $\mathrm{AD}$ symptoms came from the individual 15 minutes into the test; the test was interrupted at the patients request after 45 minutes.

Relieving the noxious stimulus by draining the bladder had an immediate impact on the $\mathrm{AD}$ symptoms, as shown in Figure 7. The release events are clearly observed in the oxygen saturation curve following an $\mathrm{AD}$ event. During $\mathrm{AD}$ oxygen content in the skin decreased to $22 \%$. Interestingly, 
a second $\mathrm{AD}$ event occurred caused by a re-filling of the bladder due to the large quantity of water consumed. During this second, less serious episode of $\mathrm{AD}$, oxygen content in the skin decreased to $42 \%$, which immediately recovered after the bladder was drained for a second time. Oxygen saturation values increased dramatically in a very brief time interval after the bladder was drained, surpassing normal values. This is indicative of a rush of oxygenated blood to the superficial capillary bed possibly causing reperfusion injury [17].

\section{CONCLUSIONS}

Autonomic dysreflexia is a fairly common event for people with spinal cord injury at or above the T6 level. Unfortunately, few SCI individuals or clinicians are aware of the potential consequences of its symptoms. The long-term effects of AD have not been explored to their full extent. One study [19] has shown that patients experiencing high levels of $\mathrm{AD}$ develop skin thickening, and since $\mathrm{AD}$ influences the circulatory system it is very possible that the skin capillary bed is also impacted. In this study we have demonstrated a quantitative method to measure the effect of $\mathrm{AD}$ on the skin oxygenation. Oxygen saturation is reduced to $40 \%$ of its original value and skin temperature is reduced of 2 to 3 degrees Centigrade possibly due to a decreased skin perfusion.

It is interesting to note that measurements of pulse oximetry in the thumb and toe of the test subject did not show any change with the onset of $\mathrm{AD}$ contrary to what was measured with the fiber probe. Pulse oximetry is a technique that measures arterial oxygenation by relying on its pulsatile nature, [33]. It is commonly accepted that the signal in pulse oximetry derives from the arterioles in the junction of dermis and hypodermis [34], this junction can be as deep as $2 \mathrm{~mm}$ in the skin and it is beyond the probing depth capability of our fiber optics (Figure 2). The signal measured in this paper comes from the nonpulsatile capillary blood flow in the dermis and papillaryplexus, part of the DC signal that is eliminated in pulse oximetry. Since the oxygen saturation values measured with the pulse oximeter did not change during $\mathrm{AD}$, we can deduce that we are measuring a local reduction in blood flow and oxygen in lieu of a systemic physiological event. The real origin of the measured signal would be better understood with a combination of reflectance spectroscopy and laser Doppler (LD) monitoring. An independent measurement of perfusion such as the one offered by the LD would clarify the impact on the signal due to a decrease in blood volume in the capillary bed as opposed to a reduction of the oxyhemoglobin molecules. Future work will be directed to such a study. Finally we want to recognize that much work remains to be done in generalizing these findings to a larger sample group, and our measurements are to be considered preliminary, but nevertheless the effect of $\mathrm{AD}$ on the circulatory system has been well documented [2-4], and a clear consequence of vasoconstriction is a peripheral decrease in perfusion, which ultimately points to skin deperfusion and deoxygenation.

\section{ACKNOWLEDGMENTS}

The authors gratefully acknowledge funding from the Christopher and Dana Reeve foundation. The authors want to thank Ted Moffit of the Oregon Medical Laser Center for providing the calibrated optical phantom. Parts of this paper have appeared in the Proceedings of SPIE-Volume 6852, Optical Fibers and Sensors for Medical Diagnostics and Treatment Applications VIII, "A fiber optic probe for measurement of an autonomic dysreflexia event on SCI patients."

\section{REFERENCES}

[1] D. Walker, Autonomic dysreflexia, B.S. thesis, University of Northampton, School of Health, Northampton, UK, 2000.

[2] A. Curt, B. Nitsche, B. Rodic, B. Schurch, and V. Dietz, "Assessment of autonomic dysreflexia in patients with spinal cord injury," Journal of Neurology, Neurosurgery \& Psychiatry, vol. 62, no. 5, pp. 473-477, 1997.

[3] A.-K. Karlsson, "Autonomic dysfunction in spinal cord injury: clinical presentation of symptoms and signs," Progress in Brain Research, vol. 152, pp. 1-8, 2005.

[4] F. U. Sack, W. Funk, F. Hammersen, and K. Messer, "Microvascular injury in skeletal muscle and skin after different periods of pressure induced ischemia," in Microcirculation and Inflammation: Vessel Wall-Inflammatory Cells-Mediator Interaction, vol. 12 of Progress in Applied Microcirculation, pp. 282288, S. Karger, Basel, Switzerland, 1987.

[5] M. Yamanaka and O. Ishikawa, "Hypoxic conditions decrease the mRNA expression of pro $\alpha 1$ (I) and (III) collagens and increase matrix metalloproteinases- 1 of dermal fibroblasts in three-dimensional cultures," Journal of Dermatological Science, vol. 24, no. 2, pp. 99-104, 2000.

[6] S. J. Dalton, D. C. Mitchell, C. V. Whiting, and J. F. Tarlton, "Abnormal extracellular matrix metabolism in chronically ischemic skin: a mechanism for dermal failure in leg ulcers," Journal of Investigative Dermatology, vol. 125, no. 2, pp. 373379, 2005.

[7] D. R. Knighton, T. K. Hunt, H. Scheuenstuhl, B. J. Halliday, Z. Werb, and M. J. Banda, "Oxygen tension regulates the expression of angiogenesis factor by macrophages," Science, vol. 221, no. 4617, pp. 1283-1285, 1983.

[8] M. Detmar, L. F. Brown, B. Berse, et al., "Hypoxia regulates the expression of vascular permeability factor/vascular endothelial growth factor (VPF/VEGF) and its receptors in human skin," Journal of Investigative Dermatology, vol. 108, no. 3, pp. 263268, 1997.

[9] D. S. Steinbrech, M. T. Longaker, B. J. Mehrara, et al., "Fibroblast response to hypoxia: the relationship between angiogenesis and matrix regulation," Journal of Surgical Research, vol. 84, no. 2, pp. 127-133, 1999.

[10] J. Thorfinn, F. Sjöberg, and D. Lidman, "Sitting pressure and perfusion of buttock skin in paraplegic and tetraplegic patients, and in healthy subjects: a comparative study," Scandinavian Journal of Plastic and Reconstructive Surgery and Hand Surgery, vol. 36, no. 5, pp. 279-283, 2002.

[11] J. Thorfinn, F. Sjöberg, L. Sjöstrand, and D. Lidman, "Perfusion of the skin of the buttocks in paraplegic and tetraplegic patients, and in healthy subjects after a short and long load," Scandinavian Journal of Plastic and Reconstructive Surgery and Hand Surgery, vol. 40, no. 3, pp. 153-160, 2006. 
[12] V. Schubert and B. Fagrell, "Local skin pressure and its effect on skin microcirculation as evaluated by laser-Doppler fluxmetry," Clinical Physiology, vol. 9, no. 6, pp. 535-545, 1989.

[13] P. G. Agache and A. S. Dupond, "Recent advances in noninvasive assessment of human skin blood flow," Acta DermatoVenereologica. Supplementum, vol. 185, pp. 47-51, 1994.

[14] F. U. Sack, W. Funk, F. Hammersen, and K. Messer, "Microvascular injury in skeletal muscle and skin after different periods of pressure induced ischemia," in Microcirculation and Inflammation: Vessel Wall_Inflammatory Cells-Mediator Interaction, vol. 12 of Progress in Applied Microcirculation, pp. 282288, S. Karger, Basel, Switzerland, 1987.

[15] E. C. Herrman, C. F. Knapp, J. C. Donofrio, and R. Salcido, "Skin perfusion responses to surface pressure-induced ischemia: implication for the developing pressure ulcer," Journal of Rehabilitation Research and Development, vol. 36, no. 2, pp. 109-120, 1999.

[16] T. Mustoe, "Understanding chronic wounds: a unifying hypothesis on their pathogenesis and implications for therapy," American Journal of Surgery, vol. 187, no. 5, supplement 1, pp. 65S-70S, 2004.

[17] T. A. Mustoe, K. O'Shaughnessy, and O. Kloeters, "Chronic wound pathogenesis and current treatment strategies: a unifying hypothesis," Plastic and Reconstructive Surgery, vol. 117, supplement 7, pp. 35S-41S, 2006.

[18] A. D. Shaw, N. Aravindan, Z. Li, and B. J. C. J. Riedel, "Temporal variation in tissue oxygen tension and perfusion response to hypoxia and ischaemia: the importance of local control of the microcirculation," The Internet Journal of Emergency and Intensive Care Medicine, vol. 7, 2003.

[19] S. L. Stover, A. M. Hale, and A. B. Buell, "Skin complications other than pressure ulcers following spinal cord injury," Archives of Physical Medicine and Rehabilitation, vol. 75, no. 9, pp. 987-993, 1994.

[20] S. L. Stover, E. F. Omura, and A. B. Buell, "Clinical skin thickening following spinal cord injury studied by histopathology," The Journal of the American Paraplegia Society, vol. 17, no. 2, pp. 44-49, 1994.

[21] R. P. Erickson, "Autonomic hyperreflexia: pathophysiology and medical management," Archives of Physical Medicine and Rehabilitation, vol. 61, no. 10, pp. 431-440, 1980.

[22] T. Moffitt, Y.-C. Chen, and S. A. Prahl, "Preparation and characterization of polyurethane optical phantoms," Journal of Biomedical Optics, vol. 11, no. 4, Article ID 041103, 10 pages, 2006.

[23] P. R. Bargo, S. A. Prahl, T. T. Goodell, et al., "In vivo determination of optical properties of normal and tumor tissue with white light reflectance and an empirical light transport model during endoscopy," Journal of Biomedical Optics, vol. 10, no. 3, Article ID 034018, 15 pages, 2005.

[24] P. R. Bargo, S. A. Prahl, and S. L. Jacques, "Optical properties effects upon the collection efficiency of optical fibers in different probe configurations," IEEE Journal on Selected Topics in Quantum Electronics, vol. 9, no. 2, pp. 314-321, 2003.

[25] N. Kollias and A. H. Baqer, "Quantitative assessment of UVinduced pigmentation and erythema," Photodermatology, vol. 5, no. 1, pp. 53-60, 1988.

[26] M. J. C. Van Gemert, S. L. Jacques, H. J. C. M. Sterenborg, and W. M. Star, "Skin optics," IEEE Transactions on Biomedical Engineering, vol. 36, no. 12, pp. 1146-1154, 1989.

[27] L. Wang, S. L. Jacques, and L. Zheng, "MCML-Monte Carlo modeling of light transport in multi-layered tissues," Computer Methods and Programs in Biomedicine, vol. 47, no. 2, pp. 131-146, 1995.
[28] T. L. Moore, M. Lunt, B. McManus, M. E. Anderson, and A. L. Herrick, "Seventeen-point dermal ultrasound scoring system-a reliable measure of skin thickness in patients with systemic sclerosis," Rheumatology, vol. 42, no. 12, pp. 15591563, 2003.

[29] T. J. Farrell, M. S. Patterson, and B. Wilson, "A diffusion theory model of spatially resolved, steady-state diffuse reflectance for the noninvasive determination of tissue optical properties in vivo," Medical Physics, vol. 19, no. 4, pp. 879-888, 1992.

[30] S. Takatani and M. D. Graham, "Theoretical analysis of diffuse reflectance from a two-layer tissue model," IEEE Transactions on Biomedical Engineering, vol. 26, no. 12, pp. 656-664, 1979.

[31] G. M. Hale and M. R. Querry, "Optical constants of water in the $200 \mathrm{~nm}$ to $200 \mu \mathrm{m}$ wavelength region," Applied Optics, vol. 12, no. 3, pp. 555-563, 1973.

[32] S. L. Jacques and D. J. McAuliffe, "The melanosome: threshold temperature for explosive vaporization and internal absorption coefficient during pulsed laser irradiation," Photochemistry and Photobiology, vol. 53, no. 6, pp. 769-775, 1991.

[33] J. F. Kelleher, "Pulse oximetry," Journal of Clinical Monitoring and Computing, vol. 5, no. 1, pp. 37-62, 1989.

[34] J. L. Reuss and D. Siker, "The pulse in reflectance pulse oximetry: modeling and experimental studies," Journal of Clinical Monitoring and Computing, vol. 18, no. 4, pp. 289299, 2004. 

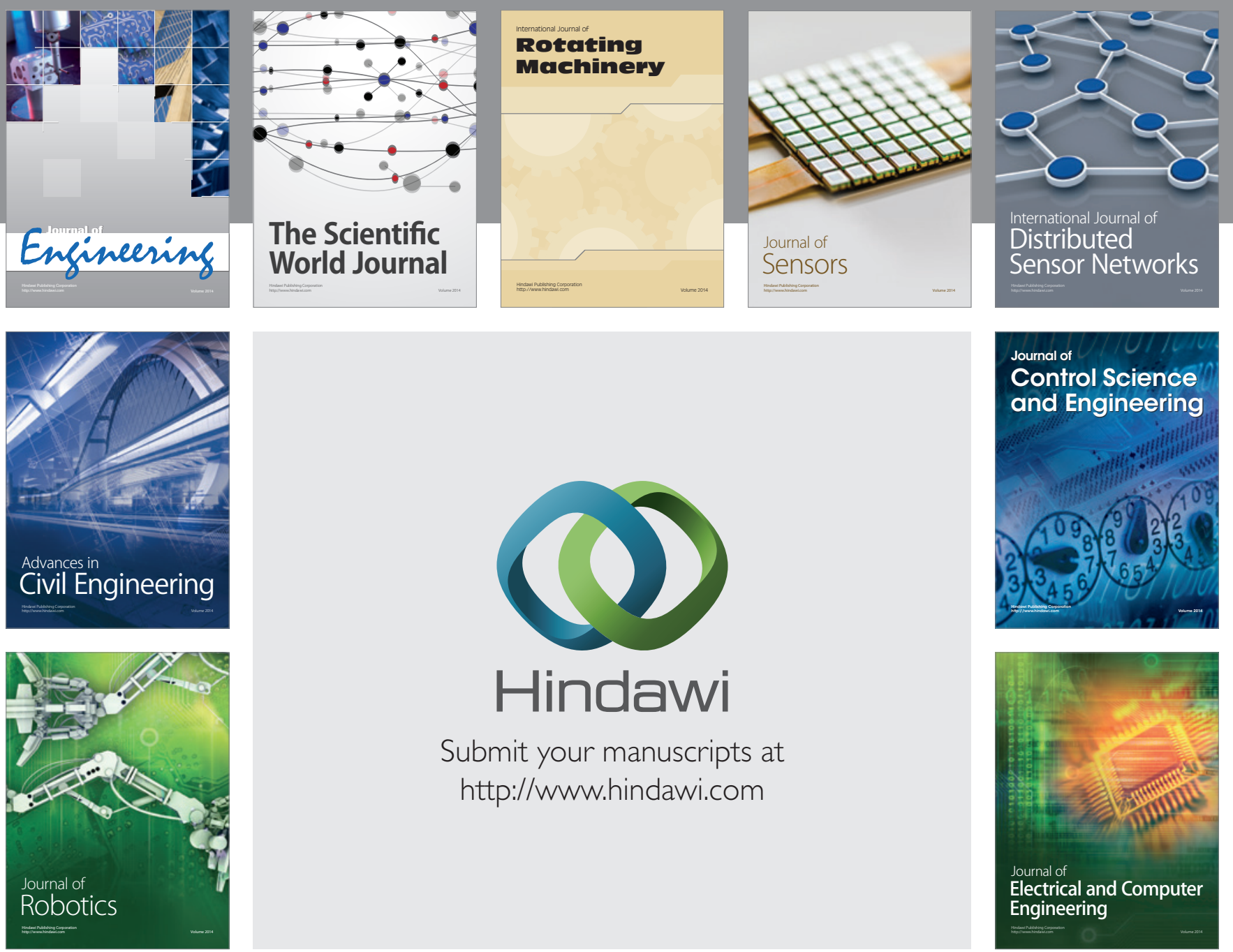

Submit your manuscripts at

http://www.hindawi.com
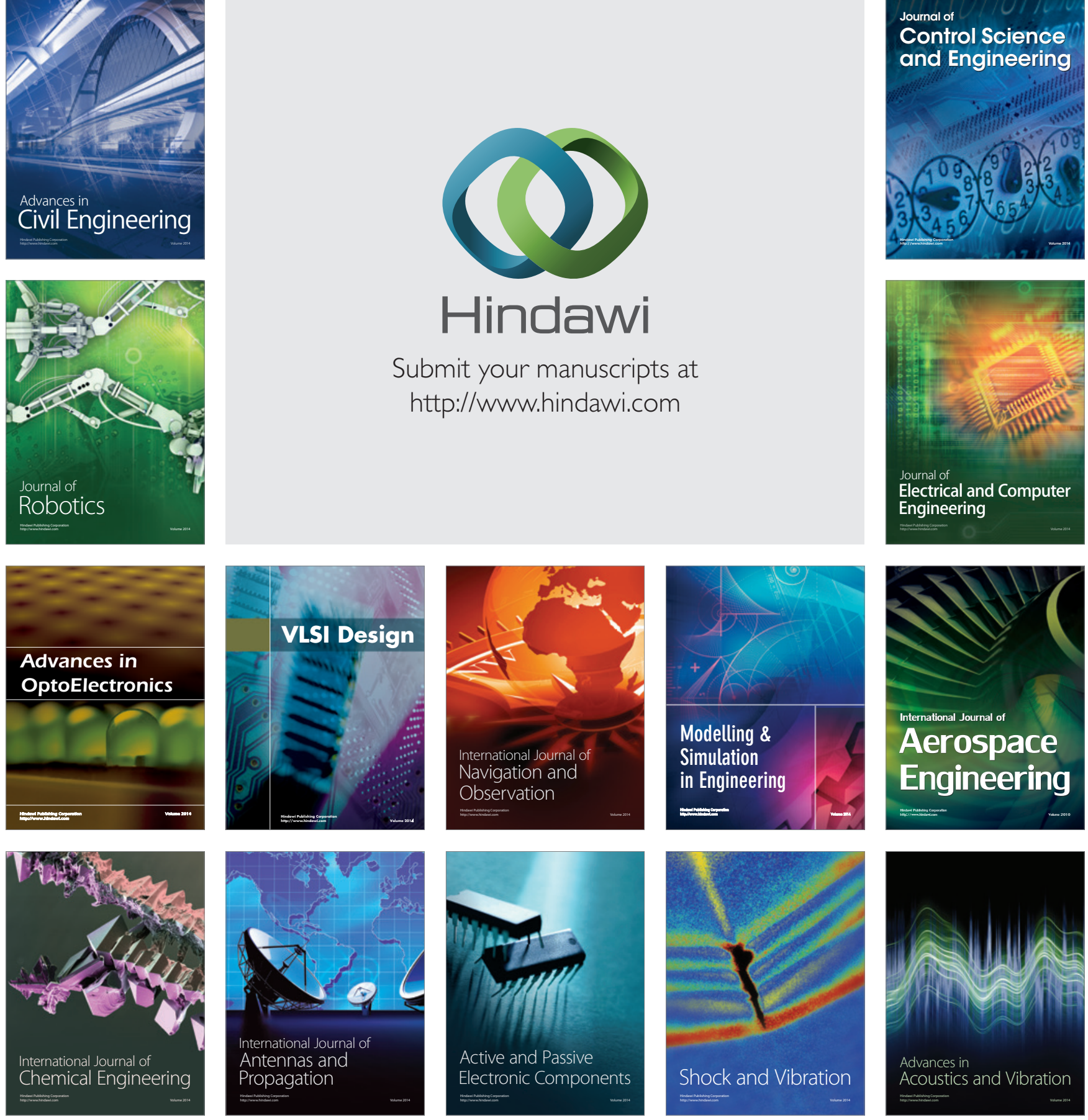